\title{
Analysis and Design of an Amplifying Circuit for Gelatinization Equipment
}

\author{
Minghui $\mathrm{You}^{1}$, Xinyu $\mathrm{Yu}^{2}$, Qixiang Sun ${ }^{1}$, Liping $\mathrm{Yin}^{1}$,Shijun $\mathrm{LI}^{1}$, \\ Xue $\mathrm{Li}^{1}$, Jingsheng $\mathrm{Liu}^{1, \text { a }}$ \\ ${ }^{1}$ Jilin Agricultural University, Changchun 130118, Jilin, China \\ ${ }^{2}$ Jilin Institute of agricultural machinery, Changchun 130021, Jilin, China \\ amhyou000@163.com
}

Keywords: the static operating point, the amplifying circuit, gelatinization equipment

\begin{abstract}
Gelatinization equipment need to resist temperature interference amplifier circuit. When the ambient temperature increases, although the collector current increases, but the partial bias amplifier circuit to make adjustments quickly to suppress the increase in the trend, making the collector current is basically the same, to ensure that the The amplifier circuit can work stably, almost independent of temperature.
\end{abstract}

\section{Introduction}

Transistor amplifier circuit, the transistor static operating point was that the AC input signal was zero. The circuit was in DC working state. The current value of the voltage can be used BJT characteristic curve of a defined point that was customarily known as the static operating point $\mathrm{Q}$ [1].The purpose of setting the static operating point was to ensure that when the amplified AC signal is added to the circuit, whether positive half-cycle or negative half-cycle could meet the transmitter forward bias, collector reverse biased transistor amplification [2].Changed the circuit parameters to change the static operating point, which could set the static operating point. If the static operating point was set improperly, saturation distortion (static operating point high) or cut-off distortion (low static operating point) occured when the AC signal was amplified[3]. The so-called static operating point, refers to the amplifier circuit was static, the circuit in which it worked state. In the Ic / Uce diagram shows a point, that was, when the determined Vcc, Rb, Rc and the transistor state of the circuit generated by the state[4]. $\mathrm{I}_{b}$ changes caused for one of the changes which were caused of the Q points along the DC load line up and down movement[5].

\section{Overall System Framework}

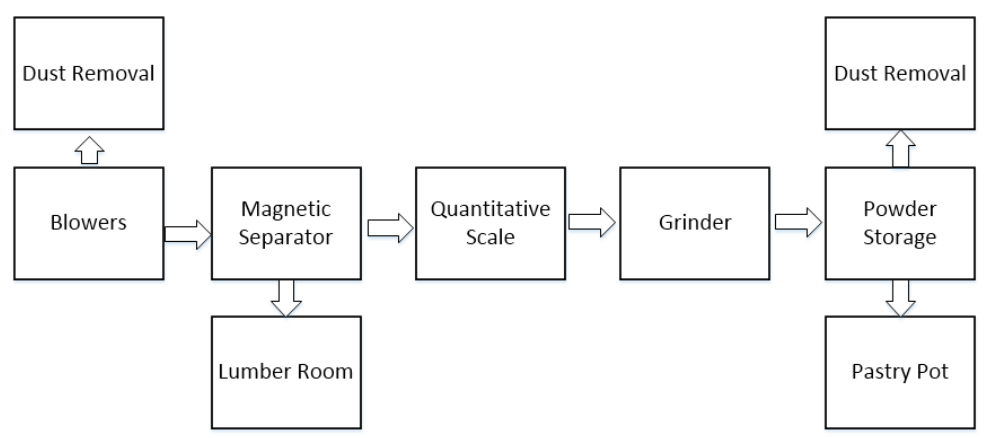

Fig1. The process associated with pasting the pot 


\section{Analysis and calculation}

\subsection{Static analysis}

Analysis of the circuit was usually calculated from the static and dynamic state of the two were discussed. We first static analysis of the circuit to calculate its static operating point.

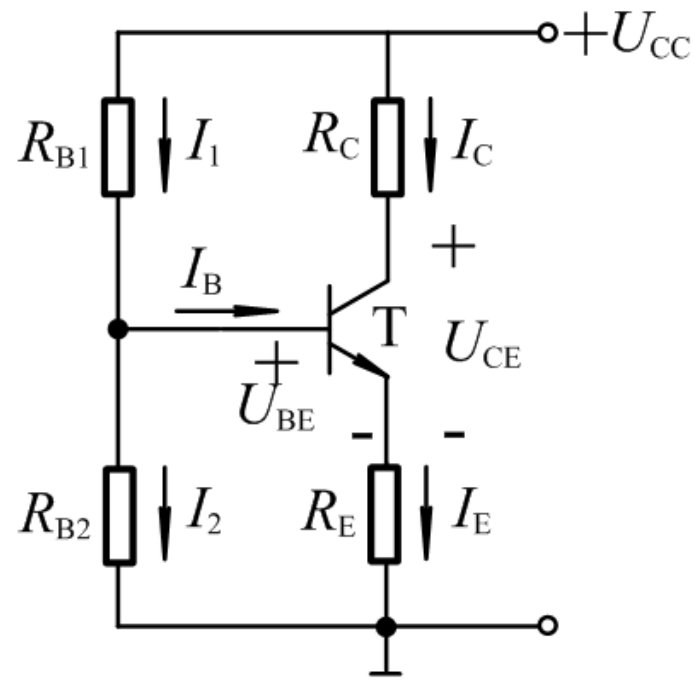

Fig1. Constant current diagram.

$$
\begin{gathered}
I_{1}=I_{2}+I_{B} \\
I_{2}>I_{B} \\
I_{1} \approx I_{2}=\frac{U_{C C}}{R_{B 1}+R_{B 2}} \\
V_{B}=R_{B 2} I_{2}=\frac{R_{B 2}}{R_{B 1}+R_{B 2}} U_{C C} \\
U_{B E}=V_{B}-V_{E}=V_{B}-R_{E} I_{E} \\
V_{B}>U_{B E} \\
I_{C} \approx I_{E}=\frac{V_{B}-U_{B E}}{R_{E}}
\end{gathered}
$$

Partial bias circuit can stabilize the static operating point of the physical process can be expressed as follows:

$$
T\left({ }^{O} C\right) \uparrow \rightarrow I_{C} \uparrow \rightarrow V_{E} \uparrow \rightarrow U_{B E} \downarrow: I_{C} \downarrow
$$

\subsection{Dynamic analysis}

The so-called dynamic analysis was to calculate the circuit in the AC voltage amplification circuit, the input resistance and output resistance. Let us first draw the communication channel. 2) For the DC power supply $U_{C C}$, because of its internal resistance was very small, the AC signal, we can put it to the AC signal, it can be used as a short circuit; Short to ground. This was the communication path we want to draw. Here was a non-linear element transistor, its existence for the 
exchange of circumstances can not be analyzed. Through the front of the learning students knew that the role of small-signal excitation could be linearized.We used it to replace the micro-equivalent circuit[6]. The transistor input $\mathrm{B}, \mathrm{E}$ equivalent between the resistance $\mathrm{r}_{\mathrm{be}}$, the output C, E equivalent between the controlled current source. This was the partial-voltage bias amplifier circuit micro-variable equivalent circuit. Next, we used this circuit on the partial bias amplifier circuit dynamic analysis[7].

\section{Summary}

Although the collector current had increased, the partial bias bias amplifier circuit made adjustments quickly to suppress the increase in the trend.Making the collector current is basically the same, to ensure that the amplifier circuit to stabilize the work.

\section{Acknowledgments}

The authors wish to express their gratitude to the projects: Jilin Province Education Department Project (No. 2015Y174), Jilin Agricultural University learning program, Jilin Provincial Department of human resources and social security Project (No. 2015Y24)for their generous support of this work.

\section{References}

[1]Xing Xiaomin.Discussion of the basic of common collector amplifier quiescent point stability. [J]Science and Technology Information.,2013,22:130-132

[2]Wei Wen.The method to fast value the performance of static state stability for transistor amplifiers. [J]Journal of EEE,2008,30(4):16-18.

[3]Ren Junyuan.Influence of quiescent points stability on dynamic performance of emitter follower. [J] Modern Electronics Technique,2013,1(1):76-78.

[4]Shen Guorong.Analysis of static operating point. [J]Journal of Liaoning Teachers College.,2000,2(3):32-36.

[5]Chen Hongbin. The necessity and method of stabilizing static operating [J]Journal of Educationnal Institute of Jilin Province,2006,22(6):72-73.

[6] Zuo Quansheng. A study on the appropriate stable bias point for transistor amplifiers [J]Journal of Changzhou Institute of Technology,2009,22(3):31-33.

[7] Dong hua.A comparative study of Chinese and foreign textbooks on DC analysis for four-resistor bias common-emitter circuit. [J]Journal of Nanchang Hangkong University:2015,29(2):104-108. 